\title{
Genetic identification and molecular characterization of Torque teno neovison virus, a novel Anelloviridae of mink in China
}

\author{
Weizhi Xin ${ }^{1}$, Zhiyuan Guo ${ }^{1}$, Lin Wang ${ }^{1}$, Lili Zhao ${ }^{2}$, Yifan Li $^{1}$, Haikun Shangguan ${ }^{1}$, \\ Chengshi Huang ${ }^{1}$, Hongyan Chen ${ }^{2}$, Mingchun Gao ${ }^{1}$, and Junwei Ge ${ }^{1}$ \\ ${ }^{1}$ Northeast Agricultural University \\ ${ }^{2}$ Chinese Academy of Agricultural Sciences Harbin Veterinary Research Institute
}

June 15, 2020

\begin{abstract}
A novel virus, designated mink Torque teno virus (mink TTV), was identified in mink. Mink TTV possesses a typical genomic organization that is consistent with those of reported anelloviruses. Phylogenetic analysis indicates that mink TTV is genetically most closely related to, but distinct from, giant panda anellovirus and swine TTV strains. This virus differs significantly from currently known members of the family Anelloviridae and represents a new species. Host-specific adaptation analysis indicates that TTVs are highly adapted to humans and primates, and observed incongruence suggests that the emergence of mink TTV was likely the result of a recent host switch from giant panda into mink. Co-infection detection indicates that mink TTV can potentially enhance the pathogenic effects of Aleutian mink disease virus and mink circovirus to cause disease.
\end{abstract}

\section{Hosted file}

Manuscript.doc available at https://authorea.com/users/333673/articles/459800-geneticidentification-and-molecular-characterization-of-torque-teno-neovison-virus-a-novelanelloviridae-of-mink-in-china 


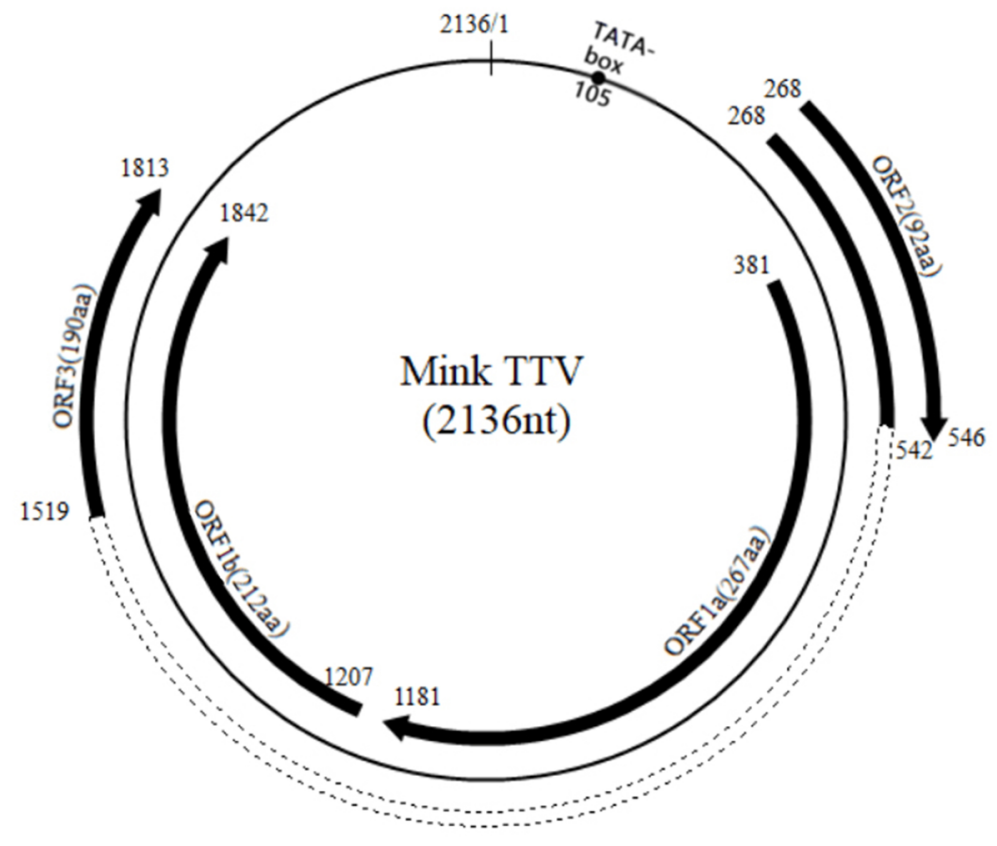

2 

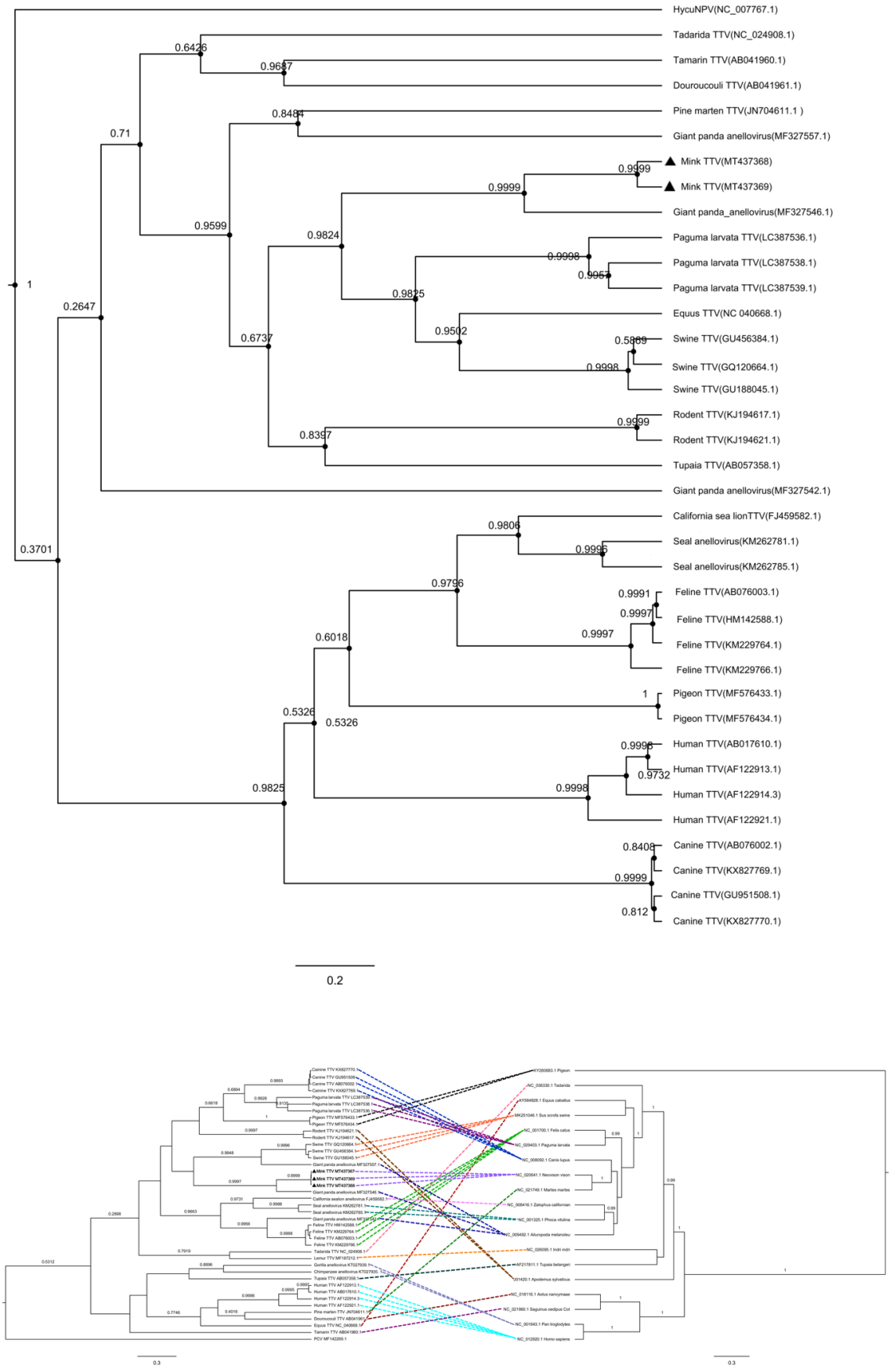


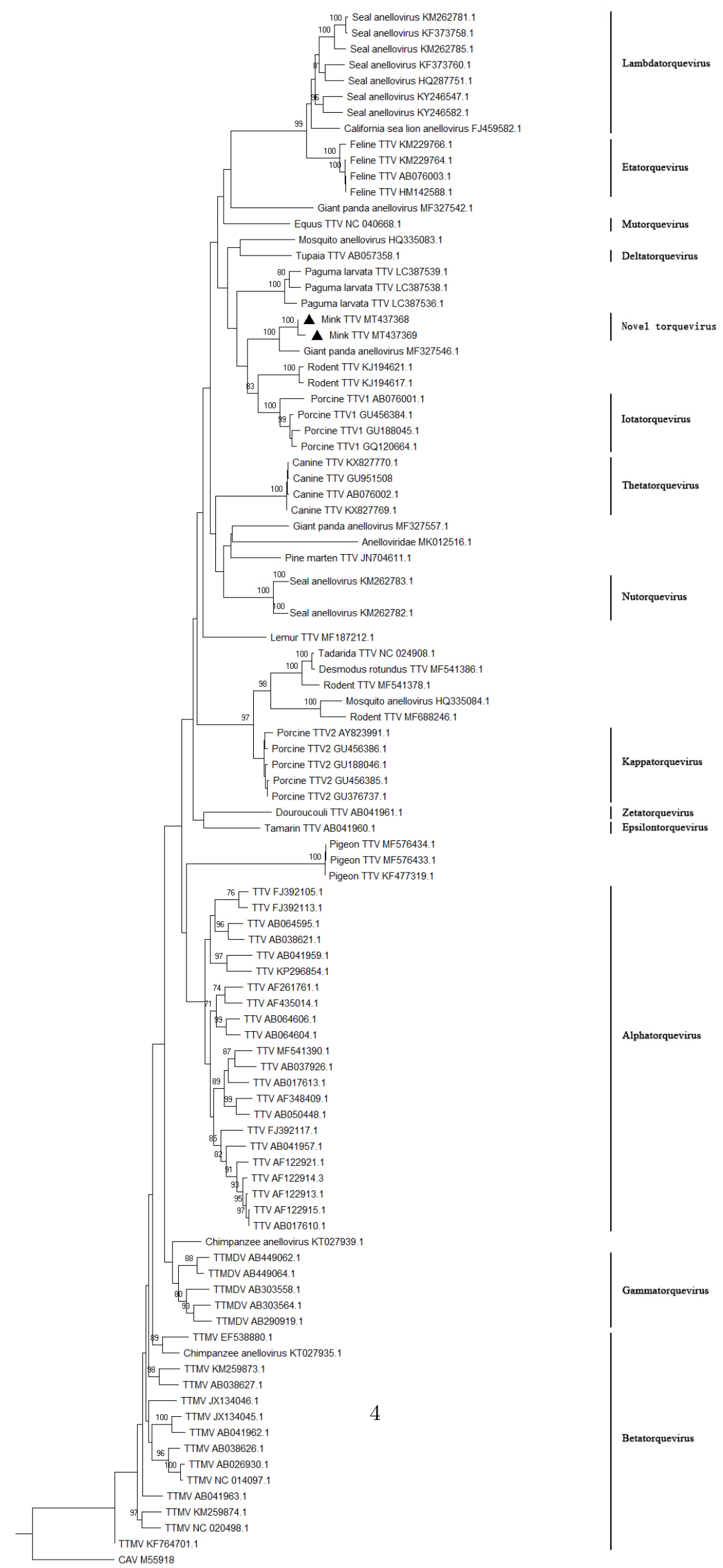

